\title{
Aggregation Mediated by Faeces and Footprints in Triatoma pseudomaculata (Heteroptera: Reduvidae), a Chagas Disease Vector
}

\section{Ana Cristina R Vitta/+ , Alicia N Lorenzo Figueiras*, Claudio R Lazzari*, Liléia Diotaiuti, Marcelo G Lorenzo}

\begin{abstract}
Laboratório de Triatomíneos e Epidemiologia da Doença de Chagas, Centro de Pesquisas René Rachou-Fiocruz, Av. Augusto de Lima 1715, 30190-002 Belo Horizonte, MG, Brasil *Laboratorio de Fisiología de Insectos, Departamento de Ciencias Biológicas, Facultad de Ciencias Exactas y Naturales, Universidad de Buenos Aires, Argentina
\end{abstract}

The behavioural response of Triatoma pseudomaculata to chemical substances present in their faeces or cuticle (footprints) was analyzed. Groups of larvae were simultaneously exposed to a clean filter paper and to another paper impregnated with a chemical stimulus in a circular arena. In these choice experiments, the insects aggregated significantly around papers impregnated with dry faeces. In addition, the bugs also showed a significant aggregation response to papers impregnated with compounds derived from their cuticle that were deposited by contact on the substrate. These results indicate that chemical compounds that affect the behaviour of T. pseudomaculata are present in the faeces and in the cuticle of this species. Results are discussed in relation to chemical communication in the Triatominae, as well as to the potential use of these substances in traps or sensors for the detection of this species.

Key words: Triatominae - Chagas disease - aggregation - behaviour - pheromones

Triatoma pseudomaculata (Corrêa \& Espínola 1964) is an haematophagous bug typically found at the Northeastern region of Brazil. It is considered a secondary vector of Chagas disease in Brazil, disease caused by the flagellate parasite Trypanosoma cruzi (Chagas 1909). It is listed among the five most important species for the transmission of this disease in Brazil. This species is predominantly found at peridomestic environments, however it may establish domestic colonies (Alencar 1987).

Previous work with T. infestans and Rhodnius prolixus demonstrated the existence of an aggregation response to volatiles present in their faeces (Schofield \& Patterson 1977, Lorenzo Figueiras et al. 1994). Lorenzo Figueiras et al. (1994) also described the dynamics of this aggregation behaviour. In that work, larvae showed a significant tendency to aggregate around papers impregnated with dry faeces. Lorenzo and Lazzari (1996) described the defecation behaviour of $T$. infestans, and demonstrated that faeces act as a chemical landmark of the refuges for this species.

In addition to the intraspecific assembling signals present in faeces (Schofield \& Patterson 1977, Ondarza et al. 1986, Lorenzo Figueiras et al. 1994), a sex pheromone released during the copula which assembles male bugs was also reported for $R$. prolixus (Baldwin et al. 1971) and

Financial support: UNDP/World Bank/WHO Special Programme of Training and Research in Tropical Disease, Capes SCyT, Universidad de Buenos Aires, Conicet, Fiocruz, Pibic programme (CNPq) for a fellowship assigned to ACRV. ${ }^{+}$Corresponding author. Fax : +55-31-3295.3115. E-mail: anavitta@cpqrr.fiocruz.br

Received 10 September 2001

Accepted 10 April 2002
T. infestans (Manrique \& Lazzari 1995).

Lorenzo Figueiras and Lazzari (1998) described another chemical signal present in the cuticle (footprints) of $T$. infestans which promotes aggregation behaviour only through physical contact. This substance was described by those authors as an arresting signal.

In this work, we investigated the existence of aggregation responses in $T$. pseudomaculata mediated by chemical substances present in the faeces or the cuticle (footprints).

\section{MATERIALS AND METHODS}

Experimental insects were 4 th instar larvae of $T$. pseudomaculata reared in laboratory at $28^{\circ} \mathrm{C}$ and fed on live chicken. They were used one week after ecdysis.

Three different experimental series were performed to study the aggregation behaviour of this species, one to test the activity of cues present in the faeces, another to test the activity of those present in the cuticle, and a third one as a control to test for any asymmetry in the arena design. In the first series, excrement was obtained by placing fed adults in plastic containers with a stainless steel mesh on the bottom. Faeces were collected on pieces of filter paper $(1.5 \times 3 \mathrm{~cm})$, placed $2 \mathrm{~cm}$ below the containers. Excrement released by bugs fell through the mesh to the container bottom, and were absorbed by the papers (Lorenzo Figueiras et al. 1994). In this way, insects never made contact with the filter papers. After $50 \mathrm{~min}$ of collection, the impregnated papers were immediately removed and maintained in a chamber at $28^{\circ} \mathrm{C}$ and $60-70 \%$ R.H. The filter papers with faeces were used six days after being collected, taking into account the data reported by Lorenzo Figueiras and Lazzari (2000).

In the second series of experiments, we tested the response of $T$. pseudomaculata to cuticular substances. Compounds present in the cuticle and deposited by con- 
tact on the substrate, i.e. footprints, were also collected on pieces of filter paper $(1.5 \times 3 \mathrm{~cm})$. The chemical stimuli were obtained as follows: a group of 15 adult insects was placed in a plastic container with 20 pieces of filter paper; the bugs, which had been starved for about 15 days and had their anus completely occluded with paraffin, to impede defecation, were allowed to walk freely on the papers (Lorenzo Figueiras \& Lazzari 1998); the papers impregnated with the substances from the cuticle were maintained inside a chamber at $28^{\circ} \mathrm{C}$ and $60-70 \%$ R.H. for nine days, and used thereafter.

The control series was performed to test if the arena and surrounding environment were symmetrical from the perspective of the insects, and therefore if their choice of a place to rest would be a random one.

All experiments were conducted in a circular glass arena (15 cm diameter) in which ten insects were liberated per assay. It was lined on the bottom with filter paper as substrate, and divided in two equal sectors. A piece of paper was placed in each sector, one impregnated with the stimulus to be tested (experimental paper) and the other clean (control paper), except for the control series in which both papers were clean. The group of larvae was carefully released in the centre of the arena. After $60 \mathrm{~min}$ the number of motionless insects in each sector was counted to measure aggregation response.

For the first series we performed five replicates, while for the second series assays were replicated six times. In the control series, six replicates were performed.

The results were analyzed statistically by means of a $\mathrm{G}$ test for Goodness of Fit to a random distribution (i.e., $50 \%$ for experimental sector and 50\% for the control).

In addition, the intensity of the aggregation response induced by each type of cue was compared using a t-test for independent samples.

\section{RESULTS}

The results obtained in the first experimental series demonstrated that the insects did not distribute in the arena by chance. Instead, a significantly greater amount of bugs assembled on or around the papers associated with the faecal signal, rather than the clean control filter papers (G-test, $P<0.005$ ). As depicted in Fig.1, approximated $70 \%$ of the experimental insects preferred the zone of the arena marked with faeces.

A similar response was observed during the second series of assays. When testing the aggregation response of the insects to papers impregnated with footprints derived substances, most insects aggregated around the experimental papers, rather than the control ones. This tendency was found to be statistically significant (G-test, $P<0.025)$. Fig. 2 shows that $65 \%$ of all bugs chose to remain in the half of the arena associated with this putative cue.

The control series of assays showed that the bugs distributed in a random manner, providing evidence that the experimental design represented a symmetrical environment for the insects (G-test, N.S.).

In order to analyse the relative relevance of these two signals for the bugs in relation to their assembling behaviour, the responsiveness of the bugs in both series was compared. No significant difference was observed when the aggregation response to faeces was compared with that evoked by footprints (t-test, N.S.). This means that the assembling response of the bugs to filter papers impregnated with their faeces or with substances from their cuticle showed a similar strength.

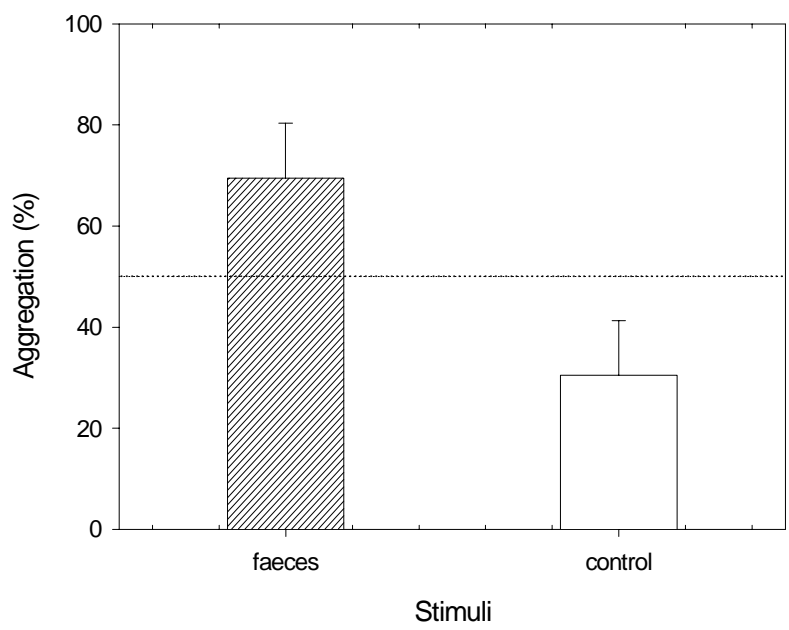

Fig. 1: aggregation behaviour in response to faeces in Triatoma pseudomaculata. Dashed line indicates the corresponding level for a random distribution (i.e. $50 \%$ of bugs at each half of the arena).

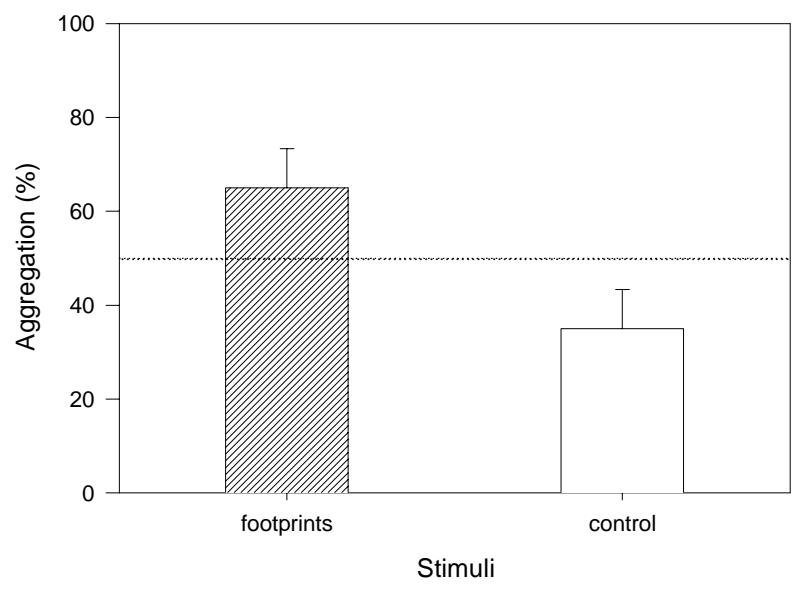

Fig. 2: aggregation behaviour in response to footprints in Triatoma pseudomaculata. Dashed line indicates the corresponding level for a random distribution (i.e. 50\% of bugs at each half of the arena).

\section{DISCUSSION}

The results presented here reveal the existence of substances in the faeces and the cuticle of T. pseudomaculata, which mediate aggregation behaviour in these bugs. This agrees with previous works that demonstrated that faeces (Schofield \& Patterson 1977, Ondarza et al. 1986, Lorenzo Figueiras et al. 1994) and cuticular substances (Lorenzo Figueiras \& Lazzari 1998) cause the aggregation of other triatomines. These findings are relevant because apparently not all triatomines employ the same communication cues, in view of some preliminary results from our labora- 
tory (Pires HHR, pers. commun.). Moreover, due to the fact that interspecific responsiveness has been evidenced for assembling cues of several triatomines, their possible use as control agents modifying the behaviour of bugs could potentially also be applicable to T. pseudomaculata.

Although aggregation cues were previously described in other triatomines, their chemical nature has not yet been elucidated. Cruz López and Morgan (1995) tested the response of T. infestans and T. mazzotti to different substances, i.e., faeces, faeces after solvent extraction and extracts. In that work, the solvent extraction was carried out using hexane, acetone, methanol or water. They showed that polar solvents remove the aggregation factor from the faeces and these solvent residues showed some positive effect. The substances present in those extracts were identified using gas chromatography-mass spectrometry (GC-MS), but bioassays using synthetic analogues of the described substances found in faeces failed to evoke aggregation responses. In addition, previously to the discovery of footprint derived substances in T. infestans (Lorenzo Figueiras \& Lazzari 1998), Juárez and Blomquist (1993) showed that cuticular compounds from T. infestans and T. mazzottii are fundamentally hydrocarbons, i.e., homologous series of n-alkanes, 3-methylalkanes and single components and isomeric mixtures of internally branched monomethylalkanes, dimethylalkanes and trimethylalkanes. Nevertheless, these authors did not test whether these substances would affect triatomine behaviour. The cuticular substance is deposited by direct contact of the insects with the substrate. Until now, the chemical nature of footprints has not been revealed, even though Lorenzo Figueiras (1997) showed that only nonpolar extracts from the cuticle evoke aggregation responses.

Although the degree of assembling induced by the two signals tested here did not differ, it does not mean that both cues involve either the same orientation mechanisms, or similar composition. It has been demonstrated in the related species $T$. infestans that faeces are able to attract distant bugs inducing positive anemotaxis, suggesting that olfactory chemoreceptors would be involved in perceiving the signal (Lorenzo Figueiras et al. 1994). On the contrary, contact chemoreceptors may be implicated in the perception of footprint derived substances, since an aggregation response was only observed when the insects were allowed to have physical contact with marked substrates (Lorenzo Figueiras \& Lazzari 1998). Concerning composition, it has been already mentioned that whereas the faecal cue showed chemical affinity with polar solvents, that of footprints eluted in non-polar ones (Lorenzo Figueiras 1997).
These two aggregation factors represent as valuable potential agents to be employed as modifiers of vector behaviour. Indeed, they appear at present as candidates to be used for population sampling or even control of triatomine bugs in human housings or wild environments. Particularly, because they are easily obtained in the laboratory from the colony and due to their interspecific activity.

\section{REFERENCES}

Alencar JE 1987. História Natural da Doença de Chagas no Estado do Ceará, Fortaleza, Imprensa Universitária da Universidade Federal do Ceará, Fortaleza, 341 pp.

Baldwin WF, Knight AG, Lynn KR 1971. A sex pheromone in the insect Rhodnius prolixus. Can Entomol 103: 18-22.

Chagas C 1909. Nova tripanosomíase humana. Mem Inst Oswaldo Cruz 1: 11-80.

Correa RR, Espínola HN 1964. Descrição de Triatoma pseudomaculata nova espécie de triatomíneo de Sobral, Ceará (Hemiptera, Reduviidae). Arq Hig Saúde Públ, São Paulo, 29: 115-127.

Cruz López L, Morgan ED 1995. Chemical investigation of aggregation behaviour of Triatoma bugs (Hemiptera: Reduviidae). J Chem Ecol 21: 2069-2078.

Juárez P, Blomquist GJ 1993. Cuticular hydrocarbons of Triatoma infestans and T. mazzotti. Comp Biochem Physiol 106B: 667-674.

Lorenzo Figueiras AN 1997. Comportamiento de Agregación y Comunicación Intra- e Interespecífica en Triatominae, $\mathrm{PhD}$ Thesis, Universidad de Buenos Aires, Buenos Aires, 160 pp.

Lorenzo Figueiras AN, Lazzari C 1998. Aggregation in the haematophagous bug Triatoma infestans: a novel assembling factor. Physiol Entomol 23: 33-37.

Lorenzo Figueiras AN, Lazzari CR 2000. Temporal change of the aggregation response in Triatoma infestans. Mem Inst Oswaldo Cruz 95: 889-892.

Lorenzo Figueiras AN, Kenigsten A, Lazzari C 1994. Aggregation in the haematophagous bug Triatoma infestans: chemical signals and temporal pattern. J Insect Physiol 40: 311316.

Lorenzo MG, Lazzari CR 1996. The spatial pattern of defecation in Triatoma infestans and the role of faeces as a chemical mark of the refuge. J Insect Physiol 42: 903-907.

Manrique G, Lazzari CR 1995. Existence of a sex pheromone in Triatoma infestans (Hemiptera: Reduviidae). I. Behavioural evidence. Mem Inst Oswaldo Cruz 90: 645-648.

Ondarza RN, Gutiérrez-Martínez A, Malo EA 1986. Evidence for the presence of sex and aggregation pheromones from Triatoma mazzotti (Hemiptera:Reduviidae). J Econ Entomol 79: 688-692.

Schofield CJ, Patterson JW 1977. Assembly pheromone of Triatoma infestans and Rhodnius prolixus nymphs (Hemiptera: Reduviidae). J Med Entomol 13: 727-734. 
Aggregation Signals of T. pseudomaculata - Ana Cristina R Vitta et al. 